\title{
Scattering of Phonons by a Vortex in a Superfluid
}

\author{
C. Wexler \\ Department of Physics, Box 118440, University of Florida, Gainesville, FL 32611-8440 \\ D. J. Thouless \\ Department of Physics, Box 351560, University of Washington, Seattle, WA 98195-1560
}

(August $10^{\text {th }}, 1998$ )

\begin{abstract}
Recent work gives a transverse force on an isolated moving vortex which is independent of the normal fluid velocity, but it is widely believed that the asymmetry of phonon scattering by a vortex leads to a transverse force dependent on the relative motion of the normal component and the vortex. We show that a widely accepted derivation of the transverse force is in error, and that a careful evaluation leads to a much smaller transverse force. We argue that a different approach is needed to get the correct expression.
\end{abstract}

67.40.Vs,67.57.Fg,47.37.+q,47.32.Cc

\section{INTRODUCTION}

Quantized vortices have been an essential part of the theory $\mathrm{g} f$ superfluids for the best part of the last few decaded. Vortices provide an important mechanism for the decay of supercurrents in either superfluids and superconductors, and their motion is responsible for dissipative effects. A considerable effort has been devoted to the derivation of the equation of motion for vortices.

It is a widely held concept that for a vortex moving with velocity $\mathbf{v}_{V}$ through a superfluid there is a force transverse to its velocity in analogy to the Magnus or Kutta-Joukowski force in classical hydrodynamics. This transverse force is given by the usual expression

$$
\mathbf{F}_{\mathrm{t}}=\rho_{s} \boldsymbol{\kappa}_{0} \times\left(\mathbf{v}_{V}-\mathbf{v}_{s}\right),
$$

where $\rho_{s}$ is the density of the superfluid component, $\boldsymbol{\kappa}_{0}$ is a vector parallel to the vortex whose magnitude is equal to the quantum of circulation $h / m$, and $\mathbf{v}_{s}$ is the superfluid velocity in the vicinity of the vortex. At zero temperature the superfluid density equals the total fluid density and this force is all there is to the story. At finite temperatures, however, part of the fluid is in the normal component and there is the possibility of additional transverse forces proportional to the velocity difference relative to this normal component, $\left(\mathbf{v}_{V}-\mathbf{v}_{n}\right)$. The existence and value of this additional transverse force has been quite controversial and verydifferent expressions for it can be found in the literature 8 .

Recent work by Thouless, Ao and Niul and Wexler unambiguously shows that the transverse force per unit length on a single quantized vortex in a superfluid is given by the universal expression in eq. 1, without any additional transverse forces. The argument is based on a global argument that relies on the equilibration of the excitations, so it does not deal separately with the change in momentum due to phonon-vortex scattering and the modification of the phonon-phonon scattering due to the vortex motion. In essence the argument is as follows:
Wexler's thermodynamic argument 5 shows that the coefficient of $\boldsymbol{\kappa}_{0} \times \mathbf{v}_{s}$ is indeed $-\rho_{s}$. This result does not appear to be controversial. Thouless, Ao and Niul showed that the part of the transverse that depends on the vortex velocity $\mathbf{v}_{V}$ is given by

$$
\left(\rho_{s} \boldsymbol{\kappa}_{s}+\rho_{n} \boldsymbol{\kappa}_{n}\right) \times \mathbf{v}_{V}
$$

where $\rho_{n}$ is the density of the normal fluid component and $\kappa_{s}, \kappa_{n}$ are the equilibrium circulations of the superfluid and normal fluid parts far away from the vortex core. The circulation of the superfluid is clearly the quantum of circulation $\kappa_{0}=h / m$ and the normalfluid is assumed irrotational $\kappa_{n}=0$ due to its viscosityl. Since the coefficients of $\mathbf{v}_{V}$ and $\mathbf{v}_{s}$ are equal and opposite Galilean invariance leaves no room left for any additional transverse force depending on the normal fluid velocity $\mathbf{v}_{n}$.

The arguments presented are rather straightforward but there is still considerable belief in the existence of additional forces. One should then consider the alternative calculations more closely and ask what is the origin of this controversy. In part, the confusion arises from different interpretations of the role played by the scattering of excitations the vortex. This is discussed next.

\section{SCATTERING OF EXCITATIONS BY A VORTEX}

There is no doubt that scattering of excitations by a vortex produces a longitudinal force proportionat to the relative velocity of the excitations and the vortex 1 . 100 - 14. For either phonons or rotons this force is quadratic in the circulation $\kappa$, and at temperatures low enough that phonons become dominant is fifth power in temperature, if only phonon-vortex scattering is taken into account and the perturbation of the phonon-phonon scattering due to the vortex motion is ignored.

It is widely believed, however, that there is also a transverse force due to an asymmetry in this phononvortex scattering. The details of this are not clear to us 
from Iordanskii's papers 13 , but a derivation by Cleary 15 which gives a transverse force linear in $\kappa$ and fourth power in temperature (like the normal fluid density dup to phonons) is widely quoted, for example by Sonins. Demircan et al.44, on the other hand, find that this force vanishes.

It has been argued 10 that the divergence of the scattering amplitude at small angles invalidates arguments, such as those in reference 14, based on the Born approximation for the scattering amplitude. In fact the $\theta^{-1}$ divergence of the scattering amplitude for small scattering angles $\theta$ gives no trouble with the longitudinal force, which depends on the scattering cross section weighted with $(1-\cos \theta)$, and only a formal divergence for the transverse force, where weighting of the differential cross section with $\sin \theta$ leads to a principle part integral and the need for a regularization procedure.

Examination of the Cleary derivation shows that the derivation is in error. Whereas Cleary obtained a transverse force per unit length proportional to

$$
\left(v_{V}-v_{n}\right) \kappa_{0} T^{4} / \hbar^{3} c^{5},
$$

a correct evaluation of the formula he used for phononvortex scattering gives an answer which term by term is of order

$$
\left(v_{V}-v_{n}\right) \kappa_{0}^{3} T^{6} / \hbar^{5} c^{9} .
$$

Cleary reached eq. 3 by adding and subtracting divergent series to his expression. Demircan et al. did not get eq. 1 because they did not keep enough terms. They did not get eq. 3 because it does not follow from Cleary's starting-point.

Let us consider a phonon in a superfluid with an infinite rectilinear vortex on the $z$-axis. We assume a stationary vortex for the calculations that follow. Galilean invariance can be used later to calculate the force in a more general situation 1 and the oscillatory motion of the vortex due to the interaction with the phonon is not important for the determination of the transverse force (see Sec. III D in Ref. 8). Phonons interact with the gaugelike superfluid velocity field and with the density variations near the vortex core. In the long-wavelength limit the former dominates 12 and phonons satisfy the following equation of motion

$$
\frac{\partial^{2} \psi}{\partial t^{2}}-c^{2} \nabla^{2} \psi+2 \mathbf{v}_{s 0}(r) \cdot \nabla \frac{\partial \psi}{\partial t}=0,
$$

where $\psi$ represents the phonon wavefunction and $\mathbf{v}_{s 0}(r)=\mathbf{e}_{\theta} \kappa_{0} /(2 \pi r)$ is the superfluid velocity around a vortex with circulation $\kappa_{0}$. Given the symmetry of the problem and the simple form of eq. 5 , it is possible to calculate the differential cross section for a phonon-vortex event exactly by using a partial-wave decomposition.

$$
\psi(\mathbf{r}, t)=\sum_{l=-\infty}^{\infty} \sum_{k_{z}} \psi_{l}(r) e^{i k_{z} z+i l \theta-i \omega t},
$$

where $\omega=c|k|=c \sqrt{k_{r}^{2}+k_{z}^{2}}$. Each partial-wave satisfies (for $l \neq 0$ )

$$
\frac{d^{2} \psi_{l}}{d r^{2}}+\frac{1}{r} \frac{d \psi_{l}}{d r}-\frac{\left(l+k \kappa_{0} / 2 \pi c\right)^{2}}{r^{2}} \psi_{l}+k_{r}^{2} \psi_{l}=0,
$$

where a small term proportional to $v_{s 0}^{2}$ has been dropped 17 . The solutions can be obtained in terms of ordinary Bessel functions $J_{\nu_{l}}\left(k_{r} r\right)$, where $\nu_{l}=\left|l+k \kappa_{0} / 2 \pi c\right|$ for $l \neq 0$ (note that from eq. 5, $\nu_{0}=0$ but since s-waves have considerable overlap with the vortex core, in general one should keep this case apart). One should also note that this is not a Born approximation solution but rather the exact solution of the phonon wave equation.

Upon consideration of the asymptotic behavior of the Bessel function one can readily obtain the scattering amplitude

$$
f(\theta)=\sqrt{\frac{2}{\pi k_{r}}} \sum_{l=-\infty}^{\infty} e^{i \delta_{l}+i l \theta} \sin \delta_{l}
$$

where

$$
\delta_{l}=\frac{\pi}{2}\left(|l|-\nu_{l}\right)=\eta \operatorname{sign}(l),
$$

and $\eta=-k \kappa_{0} / 4 c$. Note that for a vortex in superfluid helium $|\eta| \ll 1$ even for the most energetic phonons.

The series in eq. 8 are not convergent, since all the terms are of the same magnitude, but when we regularize the series in an obvious way, by writing the phase shifts as

$$
\delta_{l}=\eta \operatorname{sign}(l) \exp (-\alpha|l|), \text { with } 0<\alpha \ll 1,
$$

we get the scattering amplitude as

$$
f_{\mathrm{reg}}(\theta)=i \sqrt{\frac{2}{\pi k_{r}}} \frac{\sin \eta \cos (\eta+\theta / 2)}{\sin (\theta / 2)},
$$

in the limit $\alpha \rightarrow 0$. Here the phase shift $\delta_{0}$ has been taken to be zero. This gives the differential scattering cross section as

$$
\begin{aligned}
\frac{d \sigma}{d \theta}= & \frac{2}{\pi k_{r}} \sin ^{2} \eta\left[\cos ^{2} \eta \cot ^{2}(\theta / 2)\right. \\
& \left.-2 \sin \eta \cos \eta \cot (\theta / 2)+\sin ^{2} \eta\right] .
\end{aligned}
$$

In this form the differential cross section looks innocuous, giving a well-defined transport cross section in terms of the integral weighted with $1-\cos \theta$, and a transverse scattering cross section, in terms of the integral weighted with $\sin \theta$, that is a principal part integral. As we will see, a different limiting process can, however, give a different answer for the transverse scattering cross section, since the limiting behavior at very small angles can change the integral.

If we calculate the transverse cross section directly from eq. 8 this gives 


$$
\begin{aligned}
\sigma_{\perp} & =\int_{0}^{2 \pi} d \theta \sin \theta|f(\theta)|^{2} \\
& =\frac{2}{\pi k_{r}} \int \sin \theta d \theta \sum_{l, l^{\prime}} e^{i\left(l-l^{\prime}\right) \theta} e^{i\left(\delta_{l}-\delta_{l^{\prime}}\right)} \sin \delta_{l} \sin \delta_{l^{\prime}} \\
& =\frac{4}{k_{r}} \sum_{l} \sin \delta_{l} \sin \delta_{l+1} \sin \left(\delta_{l}-\delta_{l+1}\right)
\end{aligned}
$$

where the sums and integral were commuted to obtain the last expression. This last step is not trivial given the non-uniform convergence of the series. If the expression in eq. 14 is taken literally, then, for $\delta_{0}=0$, every term in this series vanishes. However, if the large angular momentum phase shifts are smoothly taken to zero, as in eq. 10, then the sum in eq. 14 can be expressed in terms of an integral over $\delta_{l}$ :

$$
\begin{aligned}
\sigma_{\perp} & =\frac{8}{k_{r}} \int_{\delta_{\infty}}^{\delta_{1}} \sin ^{2} \delta_{l} \sin d \delta_{l} \simeq \frac{8}{k_{r}} \int_{0}^{\eta} \sin ^{2} \delta_{l} d \delta_{l} \\
& =\frac{2}{k_{r}}(2 \eta-\sin 2 \eta) \simeq \frac{8 \eta^{3}}{3 k_{r}} .
\end{aligned}
$$

If the same integral (eq. 13) is performed for the expression for the differential cross section given in eq. 12, then we get

$$
\sigma_{\perp}=-\frac{4}{k_{r}} \sin ^{2} \eta \sin 2 \eta .
$$

These two expressions, 15 and 16, are both of order $\eta^{3}$, but differ both in sign and magnitude of the coefficient. Examination of the regularization procedure shows that in the range of angles $\alpha<<\theta<<\sqrt{\alpha}$ the even part of the scattering amplitude is proportional to $\eta^{2} \alpha / \theta^{2}$, while the odd part is proportional to $\eta / \theta$. The squares of each of these terms give contributions to the transport cross section which vanishes in the limit $\alpha \rightarrow 0$, but the product gives a contribution to the transverse cross section that is of order $\eta^{3}$. Let us look at this in more detail. We start with eqs. 8 and 9 , and assume the same regularization procedure as above, expanding this time in powers of $\eta$ while keeping $\alpha$ finite throughout the calculation:

$$
\begin{aligned}
f(\theta) & =\sqrt{\frac{2}{\pi k_{r}}} \sum_{l \geq 1} 2 i \sin \left(\eta e^{-\alpha l}\right) \sin \left(\eta e^{-\alpha l}+l \theta\right) \\
& \simeq i \sqrt{\frac{2}{\pi k_{r}}}\left[\eta \frac{\sin \theta}{\cosh \alpha-\cos \theta}+\eta^{2} \frac{\cos \theta-e^{-\alpha}}{\cosh 2 \alpha-\cos \theta}\right] .
\end{aligned}
$$

From these expressions, one can calculate the differential cross section. The square of the first term in $f(\theta)$ gives the even part of the cross section

$$
\frac{d \sigma_{e}}{d \theta}=\frac{2 \eta^{2}}{\pi k_{r}} \frac{\sin ^{2} \theta}{(\cosh \alpha-\cos \theta)^{2}}
$$

which contributes to a finite transport cross section but gives a vanishing transverse cross section $\sigma_{\perp}$. The next order term is odd in $\eta$ and $\theta$

$$
\frac{d \sigma_{o}}{d \theta}=\frac{4 \eta^{3}}{\pi k_{r}} \frac{\sin \theta(\cos \theta-\cosh 2 \alpha+\sinh \alpha)}{(\cosh \alpha-\cos \theta)(\cosh 2 \alpha-\cos \theta)},
$$

and does contribute to $\sigma_{\perp}$. If one considers the limit $\alpha \rightarrow 0$ at this point, the cross section reduces to the expression shown in eq. 12, and the transverse cross section would be given by eq. 16. The problem is that a series expansion of $d \sigma_{o} / d \theta$ in powers of $\alpha$ yields divergent contributions to $\sigma_{\perp}$ coming from small angles. The expression above, however, is completely regular for finite $\alpha$. One then calculates the transverse cross section first, which yields a regular function of $\alpha$ whose series expansion is given by

$$
\sigma_{\perp} \simeq \frac{8}{3 k_{r}} \eta^{3}\left[1-\frac{\alpha}{3}+\cdots\right] \rightarrow \frac{8 \eta^{3}}{3 k_{r}}=-\frac{k^{3} \kappa_{0}^{3}}{24 k_{r} c^{3}},
$$

in complete agreement with eq. 15. The reason for the apparent contradiction between eqs. 15 and 16 comes from important small-angle contributions which were neglected in the simple-minded approach leading to eq. 16. It is clear, however, that no contribution of order lower than $\eta^{3}$ is present.

Why does Cleary's calculation give a very different answer? In Cleary's 15 and Sonin's 8 calculations, eq. 14 was set equal to

$$
\sigma_{\perp}=\frac{1}{k_{r}} \sum_{l} \sin 2\left(\delta_{l}-\delta_{l+1}\right)
$$

which can be obtained from eq. 14 by adding and subtracting the divergent series $\left(1 / k_{r}\right) \sum \sin 2 \delta_{l}$ and $\left(1 / k_{r}\right) \sum \sin 2 \delta_{l+1}$. A result linear in $\eta$ is thus obtained from an expression that is proportional to $\eta^{3}$.

The transverse force is usually calculated by inserting the transverse cross section (eq. 20) into the phonon current (note that for this calculation we have $\mathbf{v}_{V}=\mathbf{v}_{s}=0$ ):

$$
\begin{aligned}
& \mathbf{F}_{t}^{\mathrm{ph}}=\int \frac{d^{3} k}{(2 \pi)^{3}} \sigma_{\perp}(k) n(\mathbf{k})\left(\hbar c \mathbf{k} \times \mathbf{e}_{z}\right) \\
& =-\left(\mathbf{e}_{z} \times \mathbf{v}_{n}\right) \frac{\hbar^{2}}{6 \pi^{2} k_{B} T} \int d k \sigma_{\perp}(k) \frac{k^{4} e^{\hbar c k / k_{B} T}}{\left[e^{\hbar c k / k_{B} T}-1\right]^{2}} .
\end{aligned}
$$

Without going into detail 18 it is clear that the $k^{2}$ dependence of $\sigma_{\perp}$ (eq. 20) yields a transverse force proportional to $T^{6}$ as stated in eq. 目. In contrast, the normal density is proportional to $T^{4}$ as can be clearly seen from

$\mathbf{j}^{\mathrm{ph}}=\int \frac{d^{3} k}{(2 \pi)^{3}} \hbar \mathbf{k} n(\mathbf{k}) \simeq \frac{2 \pi^{2}}{45} \frac{\left(k_{B} T\right)^{4}}{\hbar^{3} c^{5}} \mathbf{v}_{n}=\rho_{n} \mathbf{v}_{n}$.

We have seen that within this framework the result is of order $\eta^{3}$, but that the coefficient of this term depends on the limiting processes which are used. There are other reasons that make the result suspect. The first is that to the order of eq. 1 there are corrections to the simplest hydrodynamic expression for the phase shifts which 
might contribute to the same order, but which cannot possibly contribute to a lower order in $T$. The second is that that there should be contributions from the swave phase shift $\delta_{0}$, which will depend on the details of the vortex core structure. It is easy to see from eq. 14 that for $\delta_{0} \neq 0$ there is a contribution to $\sigma_{\perp}$ given by $\left(4 / k_{r}\right) \sin 2 \eta \sin ^{2} \delta_{0} \sim \kappa_{0} \delta_{0}^{2}$. While usual scattering problems in two dimensions have s-wave phase shifts that depend logarithmically on the momentum $k$, for this problem the boundary conditions are such 12 that $\delta_{0} \sim\left(k_{r} a\right)^{2}$, where $a$ is of the order of the vortex core radius. In this case the additional contribution to the transverse force will be of order

$$
\left(v_{V}-v_{n}\right) \kappa_{0}^{5} T^{8} / \hbar^{7} c^{13} .
$$

Although eq. 4 might be canceled by corrections to the hydrodynamic theory, there is no chance that eq. 24 can cancel exactly, since it is strongly dependent on the details of the core.

\section{CONCLUSIONS}

Our analysis has shown that a correct evaluation of Cleary's formula15 for the transverse scattering of phonons by a moving vortex gives a transverse force much smaller than the one he obtained, and which seems to be widely accepted. However, it is nonzero, and slightly sensitive to the details of the vortex core, which seems to contradict our claim 4 , that the transverse force on an isolated vortex is independent both of the structure of the vortex core and of the normal fluid velocity.

This is a point that requires more detailed study, but we believe that the Cleary calculation, involving the $\sin \theta$ weighted average of the differential cross section, omits the most important effect of the vortex on the phonons. This approach assumes that the incoming wave is asymptotically free, but actually for a system of radius $R$ each angular momentum mode has an energy shift $c \delta_{l} / \pi R$, and so this phase shift leads to a different equilibrium population of positive and negative $l$ modes. The shift is inversely proportional to $R$, but the number of modes contributing is proportional to $R$, so this effect is not obviously negligible 18 .

The only way we know to take this into account is to use the method which we used in Ref. 4, but with more careful examination of the boundary conditions than was given in that paper. We are working on a careful evaluation of the problem of noninteracting phonons in a moving vortex, and our preliminary results suggest that the phonons give a negative contribution close to $-\rho_{n} \boldsymbol{\kappa}_{0} \times \mathbf{v}_{V}$, which must be added to the zerotemperature result $\rho \boldsymbol{\kappa}_{0} \times \mathbf{v}_{V}$. This term comes from the negative net circulation of the phonon momentum around the vortex. This results need to be checked carefully, since it is in accord with our own preconceptions.

\section{ACKNOWLEDGMENTS}

We would like to thank Michael Stone, Edouard Sonin, Ping Ao, Xiao-Mei Zhu, Qian Niu, Lev Pitaevskii, Sandy Fetter, Alan Dorsey, Jian-Ming Tang and Jean-Yves Fortin for numerous helpful discussions. This work was supported by the NSF through grants No. DMR-9528345 and DMR-9628926.

${ }^{1}$ L. Onsager, Nuovo Cimento 6, Suppl. 2, 249-50 (1949); F. London, Superfluids II, J. Wiley, New York (1954); R. P. Feynman, in Progress in Low Temperature Physics 1, ed. C. J. Gorter, North-Holland,

${ }^{2}$ H. Lamb, Hydrodynamics, The University Press, Cambridge(1932); G. K. Batchelor An Introduction to Fluid Mechanics, Cambridge University Press (1967). Amsterdam, pp. 17-53 (1954).

${ }^{3}$ G. E. Volovik, JETP Lett. 62, 65 (1995).

${ }^{4}$ D. J. Thouless, P. Ao, and Q. Niu, Phys. Rev. Lett. 76, 3758 (1996).

${ }^{5}$ C. Wexler, Phys. Rev. Lett. 79, 1321 (1997).

${ }^{6}$ D. J. Thouless, P. Ao, Q. Niu, M. R. Geller, and C. Wexler, Proc. of IX Int. Conf. on Many-Body Physics, Sydney, 1997 (World Scientific); preprint cond-mat/9709127.

${ }^{7}$ C. F. Barenghi, R. J. Donnelly, and W. F. Vinen, J. Low Temp. Phys. 52, 189 (1983).

${ }^{8}$ E. B. Sonin, Phys. Rev. B 55, 485 (1997).

${ }^{9}$ We must note that, in general, the circulation of a viscous fluid is not necessarily zero, but keeping a non-zero circulation requires pumping energy into the system. However, in the determination of the transverse force (eq. 2), the equilibrium circulations are to be used and therefore $\kappa_{n}=0$. Aditional contributions to the transverse force, arising from a non-zero, non-equilibrium $\kappa_{n}$, are proportional to higher powers of $\mathbf{v}_{V}$.

${ }^{10}$ H. E. Hall and W. F. Vinen, Proc. Roy. Soc. (London) A238, 204 and 215 (1956).

${ }^{11}$ L. P. Pitaevskii, Zh. Eksperim. i Teor. Fiz. 35, 1271 (1958) [Sov. Phys. JETP 8, 888 (1959)].

12 A. L. Fetter, Phys. Rev. 136, A1488 (1964).

13 S. V. Iordanskii, Zhur. Eksp. Teor. Fiz. 49, 225 (1965) [Sov. Phys. JETP 22, 160 (1966)].

${ }^{14}$ E. Demircan, P. Ao and Q. Niu, Phys. Rev. B 52, 476 (1995).

${ }^{15}$ R. M. Cleary, Phys. Rev. 175, 587 (1968).

16 A. Shelankov, preprint cond-mat/9802158.

17 See references 1 and 18 for corrections to the hydrodynamic expressions. Also see the discussion immediately after eq. 23

18 For further discussion, see C. Wexler, Ph.D. thesis, University of Washington, 1997. 